Original Article

\title{
ANTIMICROBIAL POTENTIALS OF SOIL FUNGI METABOLITES, MOLECULAR DYNAMICS AND THEIR 3D PROTEIN STRUCTURAL PREDICTION USING BIOINFORMATICS TOOL
}

\author{
MAJOLAGBE 0. N. ${ }^{1 *}$, AINA D. A. ${ }^{2}$, OMOMOWO I. 0.1, THOMAS A. ${ }^{1}$
}

${ }^{1}$ Microbiology Unit, Department of Pure and Applied Biology, Ladoke Akintola University of Technology, PMB 4000, Ogbomoso Oyo State, Nigeria, ${ }^{2}$ Department of Microbiology, Babcock University, Ilishan-Remo, Ogun State

Email: oomajolagbe@lautech.edu.ng

Received: 16 Jun 2020, Revised and Accepted: 14 Aug 2020

\begin{abstract}
Objective: To determine the antimicrobial potentials of secondary metabolite of soil fungi and predict their 3D structure and molecular identity.

Methods: Pure soil fungi were isolated from soil samples and cultured under submerged fermentation (Smf) for their metabolites using Potato Dextrose Agar and Broth. The secondary metabolites of the isolated fungi were obtained intracellularly after $21 \mathrm{~d}$ of incubation in a rotary shaker incubator. The antimicrobial potentials of the metabolites were investigated against four (4) clinical isolates, namely: Staphylococcus aureus, Klebsiella spp, Candida albicans and Escherichia coli. These soil fungi were further characterized to the molecular level and their evolutionary relationships established using bioinformatics tools. Protein structure of each of the fungi isolates was predicted using PHYRE-2.

Results: Out of all the soil fungi isolated, the metabolite of Aspergillus aculeatus showed the highest antimicrobial activities against Staphylococcus aureus $(23.00 \pm 2.34 \mathrm{~mm})$, Escherichia coli $(9.00 \pm 1.44 \mathrm{~mm})$ and Klebsiella spp $(24.00 \pm 3.45 \mathrm{~mm})$. The 3D protein structure predicted showed that each of the organisms consists of different amino-acid compositions such as: serine, tyrosine, proline, arginine, glycine, phenylalanine leucine with other notable biological properties.
\end{abstract}

Conclusion: The work revealed that secondary metabolites of the isolated fungi carry an important role in combating infectious agents thereby, providing roadmaps for the biosynthesis of many synthetic and semi-synthetic drugs and bio-products which are environmentally friendly.

Keywords: Secondary metabolite, Antimicrobial, Soil fungi, Molecular dynamics

(C) 2020 The Authors. Published by Innovare Academic Sciences Pvt Ltd. This is an open access article under the CC BY license (http://creativecommons.org/licenses/by/4.0/) DOI: http://dx.doi.org/10.22159/ijcpr.2020v12i5.39759. Journal homepage: https://innovareacademics.in/journals/index.php/ijcpr

\section{INTRODUCTION}

Kingdom Fungi is the second largest kingdom after insects [1]. They are found all over the planet, even in the most hostile and uninhabitable places such as in Antarctic ice, mountain surfaces, rocks, seawater and habitable places like the tropics and temperate regions [2]. Fungi possesses arays of many chemical molecules with biochemical activities. They produce several types of enzymes and secondary products of metabolism, which include antibiotics, vitamins, polysaccharides and organic acids [3]. Fungi play very important roles in human daily life, which ranges from its use in many industrial processes to agriculture, medicine, food processing, textile, biomedical production, natural cycling, and production of bio-fertilizers and so on. Therefore, fungal biotechnology has become a very important part of human welfare [4]. Fungi are fastgrowing organisms that do well on the surface of most plant and other organic substances. They have adapted to survive on many substrate in other to survive, reproduce and compete with other life forms. Fungi have divers mood of nutrition such as symbiotic, parasitic and saprophytic. Apart from humans, fungi also play a beneficial and necessary part in the survival and growth of many plants by suppressing plant root diseases, protecting the plants from pathogens; they also improve their host plant ability to acquire nutrients by antagonism through the production of plant repellant. Fungi also provide for their host plant many vitamins needed for plant growth. Some of the metabolite produced by fungi within a plant host increases the plant ability to withstand the invasion of predators, parasites and diseases and may facilitate reproductive processes. One of the known characteristics of fungi is their nature as food spoilers that causes damage to raw and cooked food, some species of fungi are plant pathogens such as the causative organism of Potato late blight caused by phytophthora infestans, which was responsible for the Potato famine of Ireland in 1845-1849, and rice blast which caused the infamous Bengal famine of 1943. The fungi ergot formed by mycelium of Claviceps spp especially C. purpurea have been implicated for the poisoning of cattle. The mycotoxins aflatoxins and ochratoxin A produced by many species of Apergillus are a common cause of food poisoning in many countries. Nevertheless, fungi have been found to be beneficial to man as producers of many different metabolites such as antibiotics, enzymes such as cellulase, lipase and ligninolytic enzymes). They are also known for the production of alkaloids such as ergot alkaloids from Claviceps, pigments such as anthraquinone and betalains. Fungi have also been reported in biological control of nematodes, also are good sources of aroma and flavors. Some edible fungi are also a source of nutrition as they provide essential elements such as selenium, potassium, riboflavin, niacin and vitamin D. In addition, edible fungi can also prevent or treat some diseases such as Parkinson, Alzheimer, hypertension, cancer and stroke [2, 5]. Funi could perform these functions due to array of metabolites which they possess such as: esters, aldehydes, aromatic compounds, organi acids, etc [6].

\section{MATERIALS AND METHODS}

\section{Soil samples collection}

Soil samples were collected at various locations in the Horticultural garden of LAUTECH and then taken to Microbial Resources and Research Laboratory, Ogbomoso. This was serially diluted and inoculated onto a prepared Potato Dextrose Agar (PDA) plate containing antibacterial (Chloramphenicol) to inhibit bacteria growth on the plate with one plates serving as control. They were incubated at $28^{\circ} \mathrm{C}$ for $24-72 \mathrm{~h}$. pure fungi isolates were obtained by sub-culturing. The Potato Dextrose Broth (PDB) was prepared by dissolving $24 \mathrm{~g}$ in 1liters of distilled water, it was mixed and dissolves by heating with frequent agitation, and it was boiled for some minutes until complete dissolution is obtained according to the manufacturer's guidelines. Then it was dispensed into appropriate bottle, $500 \mathrm{ml}$ in eight places, sterilized in an autoclave at $121^{\circ} \mathrm{C}$ for $15 \mathrm{~min}$. 


\section{Submerged fermentation ( $\mathrm{smf}$ ) of soil fungi isolates}

Submerged fermentation (smf) was done using Potato Dextrose Broth (PDB). The eight isolates were coded as OT, AS, OS, GT, AT, GS, $\mathrm{BT}, \mathrm{BS}$ and were inoculated into the broth medium in a $500 \mathrm{ml}$ Erlenmeyer flasks. All experiments were carried out aseptically inside laminar air-flow chamber. The flasks were placed in a rotaryshaker incubator at $28{ }^{\circ} \mathrm{C}$ for seven (7) days while biomass and metabolite production were moniutoter daily.

\section{Extraction of the fungi metabolite}

The organisms produced their metabolites intracellularly-that is within its cells. Extraction of the metabolites was performed after 21 $\mathrm{d}$ of culture. The biomass was filtered using sterile muslin cloth (sterilized in an autoclave at $121{ }^{\circ} \mathrm{C}$ for $15 \mathrm{~min}$ ) while the metabolites were extracted using Ethanol at ratio 2:1 of the culture media. The metabolites were obtained after centrifugation at 5,000 $\mathrm{rpm}$ for $10 \mathrm{~min}$. The metabolites were obtained for each of the soil fungus andf then kept at $4{ }^{\circ} \mathrm{C}$ for turther use.

\section{Molecular characterization of the soil fung}

\section{Fungal genomic DNA extraction}

Fungal isolates were grown on cellophane membrane fixed on Potatoe Dextrose Agar and were incubated at $25{ }^{\circ} \mathrm{C}$. The fungal isolates weighing about $50-100 \mathrm{mg}$ (wet weight) were placed in a sterile ZR Bashing Lysis Tubes containing $750 \mu \mathrm{l}$ of lysis buffer, the mixture was put in a bead fitted $2 \mathrm{ml}$ tube holder assembly and crushed at the highest speed of $>5$ min, the preparation was swirled briefly in the ZR Bashing Bead ${ }^{\mathrm{TM}}$ Lysis Tube then centrifuged at $10,000 \mathrm{rpm}$ for one minute a micro-centrifuge. Resultant supernatant of about $400 \mu \mathrm{l}$ was transferred into a Zymo-Spin ${ }^{\mathrm{TM}} \mathrm{IV}$ Spin Filter (orange top) in a collection tube and centrifuged for one minute at 7,000rpm, a $1200 \mu \mathrm{l}$ measure of fungal DNA binding buffer was added to the filtrate in the collection tube. Then, $800 \mu \mathrm{l}$ of the mixture was transferred to a Zymo-Spin ${ }^{\mathrm{TM}}$ IIC Column in a Collection Tube and centrifuged 10,000 rpm for 1 minute period. The flowthrough was discarded then, $200 \mu \mathrm{l}$ DNA pre-washed buffer was added to the Zymo-Spin ${ }^{\mathrm{TM}}$ IIC Column in new collection tube; this was centrifuge at $10,000 \mathrm{rpm}$ for 1 minute. After centrifugation 500ul fungal DNA wash buffer was added to a Zymo-Spin TM IIC Column and centrifuged at 10,000rpm for one minute. Zymo-Spin TM IIC Column was transferred to a clean $1.5 \mathrm{ml}$ micro-centrifuge tube and $100 \mu \mathrm{l}$ (35 $\mu \mathrm{l}$ minimum) DNA elution buffer was added directly to the column matrix and centrifuged at 10,000 rpm for 30 seconds to elute the fungal DNA. DNA was then suitable for PCR and other downstream applications.

\section{Polymerase chain reaction (PCR) of the extracted fungal DNA}

The extracted fungal DNA were amplified in a thermal cycler (Gene AMP PCR system 9700); the PCR reaction mixture consisted of $190 \mathrm{u}$ extracted fungal DNA, 2.5ul 10x PCR loading buffer, $2.0 \mathrm{ul} 2.5 \mathrm{Mm}$ DNTPs mix, $1.0 \mathrm{ul} 25 \mathrm{Mm} \mathrm{mgcl}_{2}, 1 \mathrm{ul}$ DMSO, $5 \mathrm{ul}$ of taq polymerase and $1 \mathrm{ul}$ each of the forward and reverse primer:

ITS1F: 5'-TCCGTAGGTGAACCTGCGG-3' and

ITS2R: 5'-GCTGCGTTCTTCATCGATGC-3'

\section{Antimicrobial susceptibility test}

Four (4) clinical isolates namely: Staphylococcus aureus, Klebsiella spp, Candida albicans and Escherichia coli isolated from clinical samples at LAUTECH, Ogbomoso were used to test for the antimicrobial activities of the metabolites (the metabolites were tagged as: OT, AS, OS, GT, AT, GS, BT, BS). Muller Hilton agar was prepared and poured into Petri dishe. After it has cooled down, the clinical isolates were evenly streaked onto the surface of the plate. $100 \mu \mathrm{l}$ of each of the metabolites was impregnated onto a paper filter paper disc and then placed on the center of the plates. All the eight (8) metabolites were tested against the four (4) clinical isolates. They were incubated for $18-24 \mathrm{~h}$ at $37{ }^{\circ} \mathrm{C}$. The inhibitory activities of the metabolites against the pathogenic organisms was determined and measured in $\mathrm{mm}$

\section{Protein structure and function of the fugal isolates}

After the DNA nucleotide sequences were obtained, the sequence was transferred to European Molecular Biology open software suit (EMBOSS) to translate the sequence into amino acid. The amino acid sequence was transferred to PHYRE 2 for the possible protein prediction and viewed using PYMOL software.

\section{RESULTS}

\section{Isolation and prevalence soil fungi}

The soil samples were collected inside foil papers using spatula to dig through the soil at a depth of $1-5 \mathrm{~m}$. Serial dilution was performed for the fugi isolation. The diluent of $10^{-3}$ and $10^{-4}$ test tubes was inoculated into the prepared Potato Dextrose Agar (PDA) plate containing antibacterial (Chloramphenicol) to inhibit bacteria growth on the plate. The plates were incubated at $28{ }^{\circ} \mathrm{C}$ for about 3 d. Growth pattern was observed from the third day. The cultures were transferred onto slant bottles for biochemical characterization and identification. (Plates 1-2). In this study, isolates of the Aspergillus genus had the highest occurrence $(62.5 \%)$ followed by Aspergillus aculeatus (50\%), Aspergillus Carbonarius (12.5\%) followed by Penicillium genus $(25 \%)$ Penicillium verrucosum (12.5\%), Penicillium chrysogenum (12.5\%) and Talaromyces albobiverticillius (12.5\%).

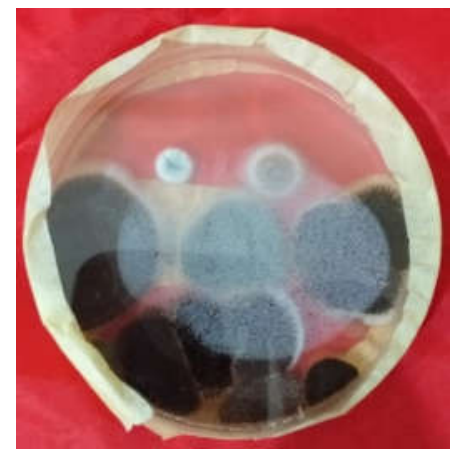

Plate 1: Culture of soil fungi on PDA plate

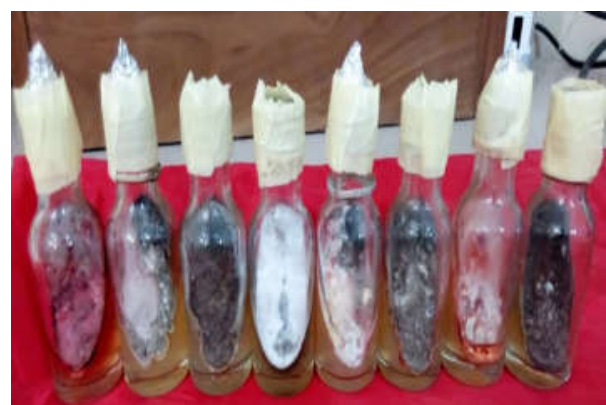

Plate 2: Cultures of soil fungi preserved on PDA slants

Molecular identification and evolutionary relationship of soil fungi

The nucleotide sequences obtained were blasted at the National Center for Biotechnological Information (NCBI) Genbank. FASTA formats were obtained of other related sequences and used to plot a phylogenetic tree that established the evolutionary relationship with the query sequence (fig. 1-7). The soil fungal isolates were identified to be: Penicillum verrucosum, Talaromyces albobiverticillus and Aspergillus spp which were A. aculeatus and carbonarius (table 1 ). 
Table 1: Soil fungi identity



Fig. 1: Phylogenetic relationship of LAU-MAJ-ASF and Pencillium species

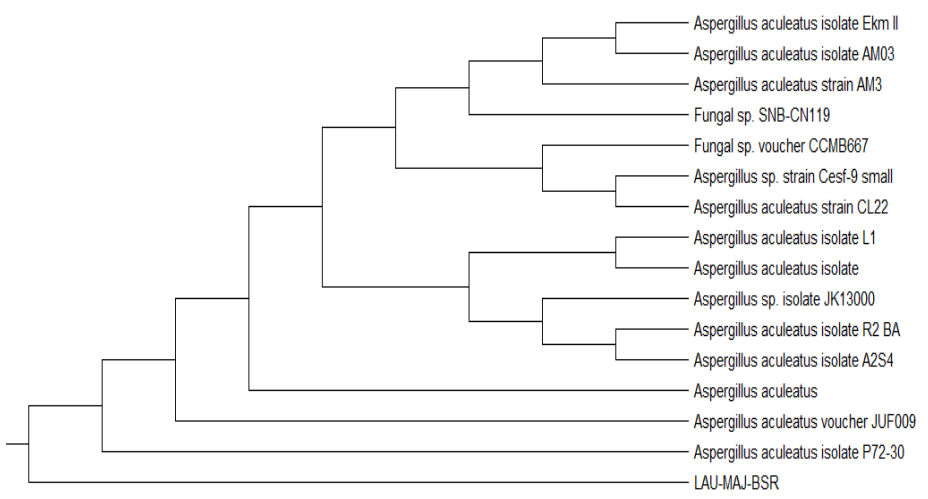

Fig. 2: Phylogenetic relationship of LAU-MAJ-BSR and Aspergillus species

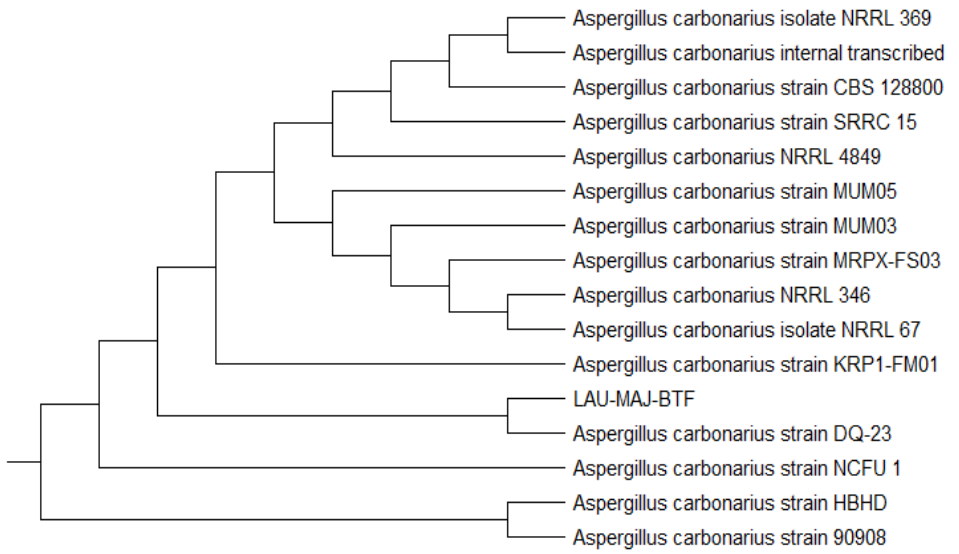

Fig. 3: Phylogenetic relationship of LAU-MAJ-BTF and Aspergillus species 
The evolutionary history of the isolates was determined by UPGMA [7]. The sum of the branch length of the optimal tree was shown to be 0.61519578. Evolutionary distances were determined using the maximum composite method $[8,9]$. The analyses include 16 nucleotide sequences. Every ambiguous positions were deleted for each sequence pair. A total of 628 positions were recorded for the final dataset. Evolutionary analyses were conducted in MEGA X [9].



Fig. 4: Phylogenetic relationship of LAU-MAJ-GSF and Talaromyces species

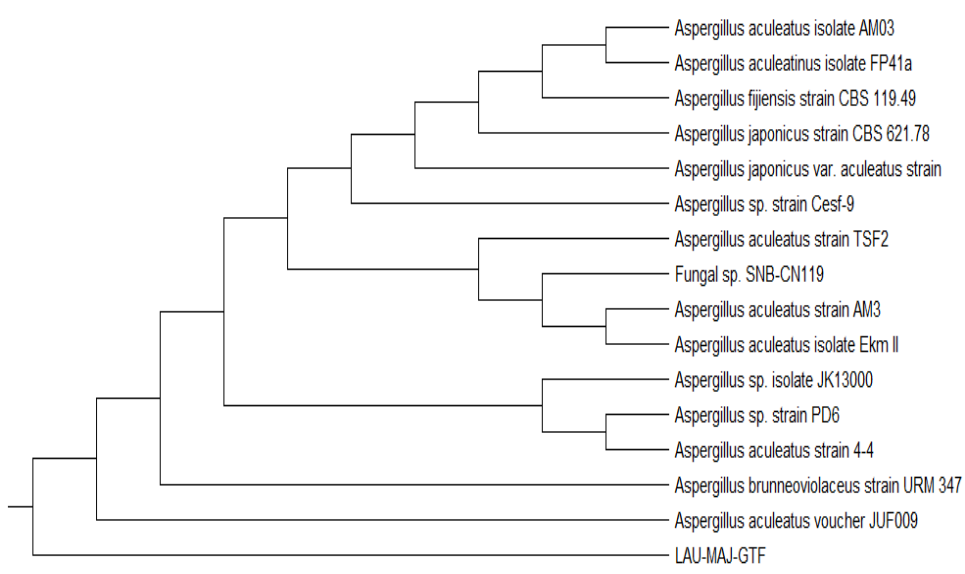

Fig. 5: Phylogenetic relationship of LAU-MAJ-GTF and Aspergillus species

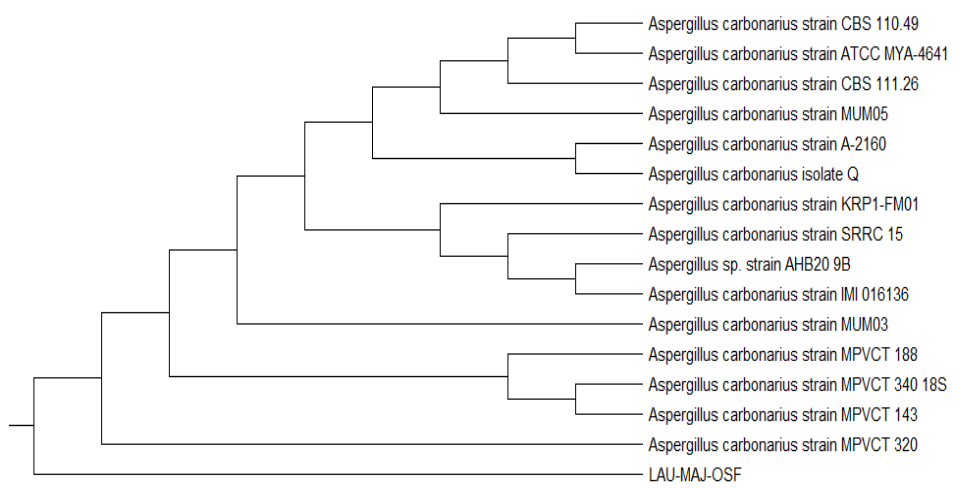

Fig. 6: Phylogenetic relationship of LAU-MAJ-OSF and Aspergillus species

\section{Protein sequence of metabolite}

After the DNA nucleotide sequence was obtained, the sequence was transferred to European Molecular Biology Open Software Suite (EMBOSS) to translate the sequence into amino acid. The amino acid sequence was transferred to PHYRE 2 software that was used to model the 3D protein structure and viewed with PYMOL Software (Plate 3a-h). In terms of functions, the proteinns were found to be structural protein, proton transporter, oxidoreductase and hydrolase/hydrolase inhibitors. 


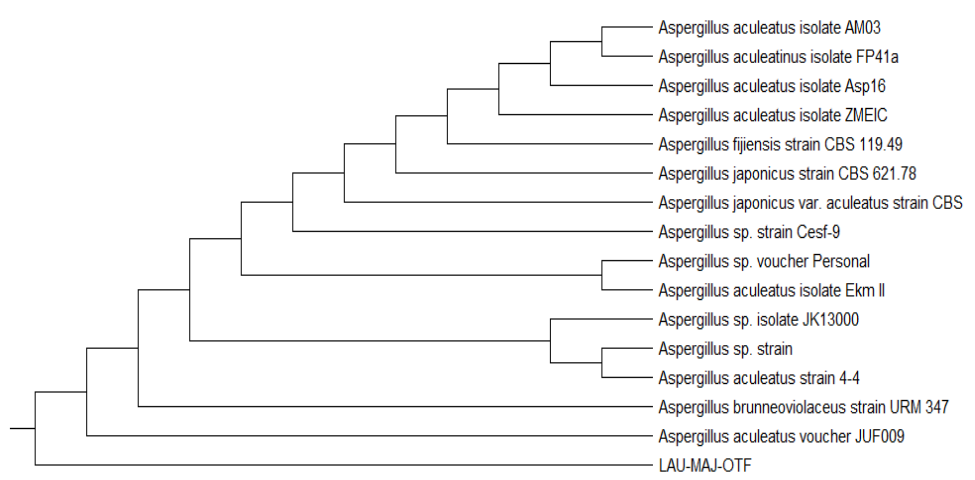

Fig. 7: Phylogenetic relationship of LAU-MAJ-OTF and Aspergillus species

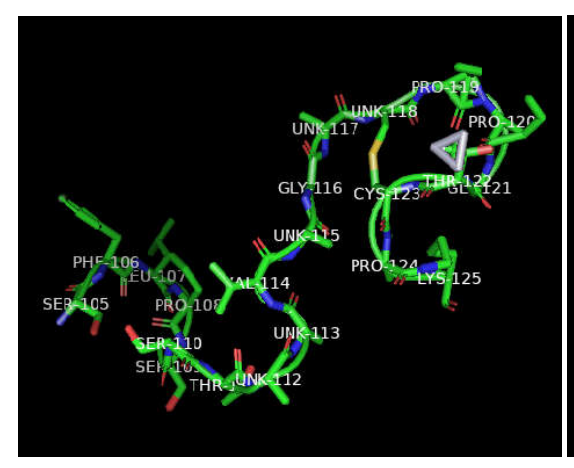

3(a)

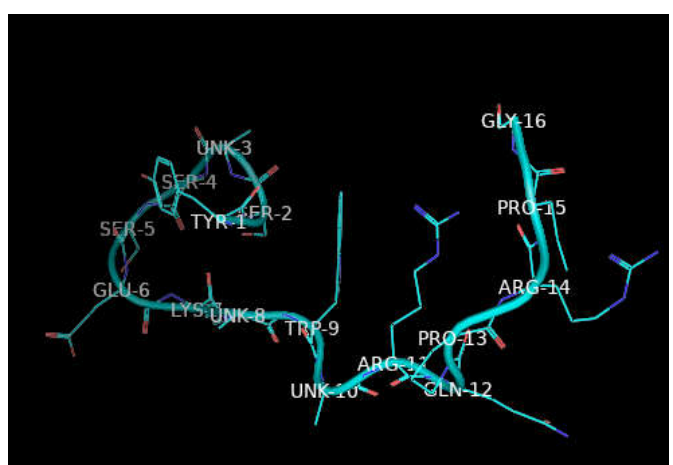

$3(c)$

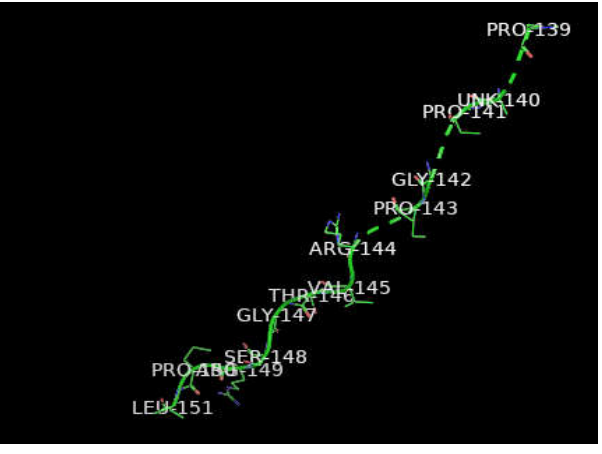

3(b)

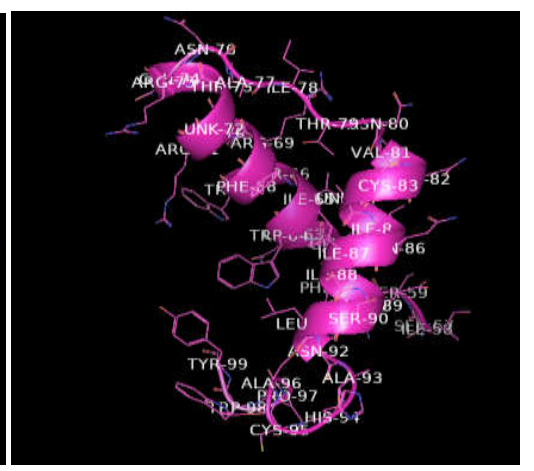

3(d)

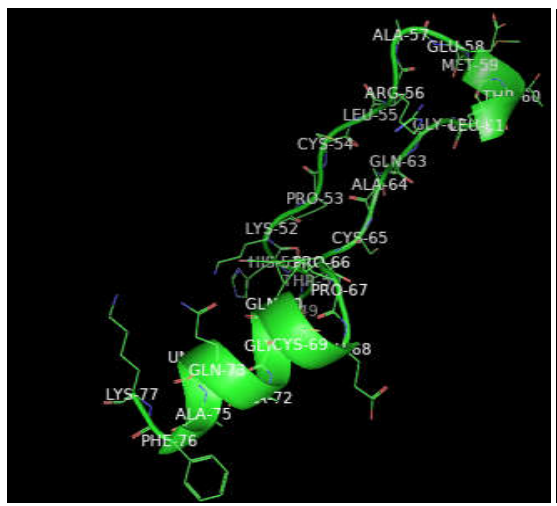

3(e)

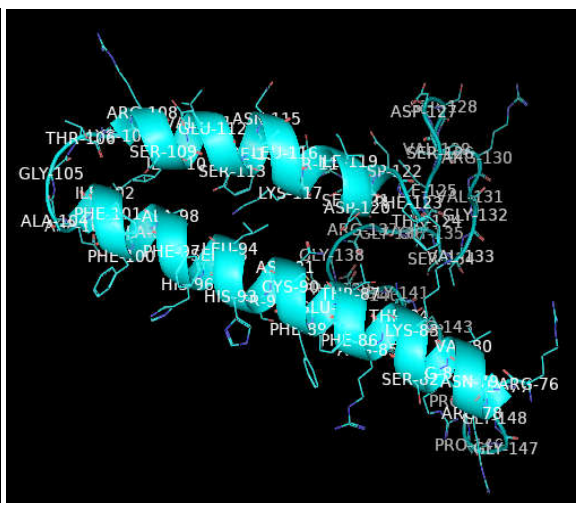

3(f)

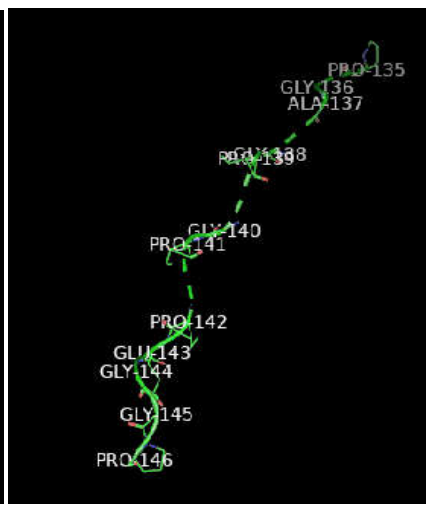

$3(\mathrm{~g})$

Plate 3 (a-g) Modelled 3D Protein structure (a-Penicillum verrucosum; b-Penicillium chrysogenum; c-Aspergillus aculeatus; d-Aspergillus carbonarius; e-Talaromyces albobiverticillius; f-Aspergillus aculeatus; g-Aspergillus aculeatus) showing the amino acid residues 
Table 1: Zone of inhibition by the soil fungi metabolites against clinical isolates

\begin{tabular}{|c|c|c|c|c|}
\hline \multirow[t]{2}{*}{ Fungi metabolites } & \multicolumn{4}{|c|}{ Zone of inhibition in diameter (mm) } \\
\hline & Staphylococcus aureus & Escherichia coli & Candida albicans & Klebsiella spp \\
\hline Penicillum verrucosum(AS) & - & - & - & - \\
\hline Penicillium chrysogenum (AT) & - & - & - & - \\
\hline Aspergillus aculeatus (BS) & $23 \pm 0.55$ & $9 \pm 0.47$ & - & $24 \pm 2.45$ \\
\hline Aspergillus carbonarius(BT) & $21 \pm 2.67$ & - & - & - \\
\hline Talaromycesalbobiverticillus(GS) & - & - & - & - \\
\hline Aspergillus aculeatus(GT) & - & - & - & - \\
\hline Aspergillus carbonarius(OS) & - & - & - & - \\
\hline
\end{tabular}

Resistant (-)

\section{Determination of zones of inhibition against clinical isolates}

The antimicrobial activities of the metabolite of the eight (8) isolates were carried out against four (4) pathogenic organisms of clinical origins namely: Staphylococcus aureus, Escherichia coli, Candida albicans and Klebsiella spp. Out of all the metabolite tested against the pathogens, the metabolite of Aspergillus aculeatus (BS)showed the highest and prominent inhibition against $S$. aureus $(23 \pm 0.55 \mathrm{~mm})$, E. coli $(9.00 \pm 0.47$ $m m)$, Klebsiella spp (24.00 $\pm 2.45 \mathrm{~mm}$ ) but no zone of inhibition against $C$. albicans. Metabolite of $A$. carbonarius had a zone of inhibition of $21 \pm 2.67$ $\mathrm{mm}$ against $S$. aureus but no zone of inhibition against $C$. albicans, Klebsiella spp and E. coli. The clinical isolate showed resistance to metabolites of Penicillium verrucosum, Penicillium chrysogenum, Talaromyces albobiverticillius and Aspergillus aculeatus (GT) as presented in table 1.

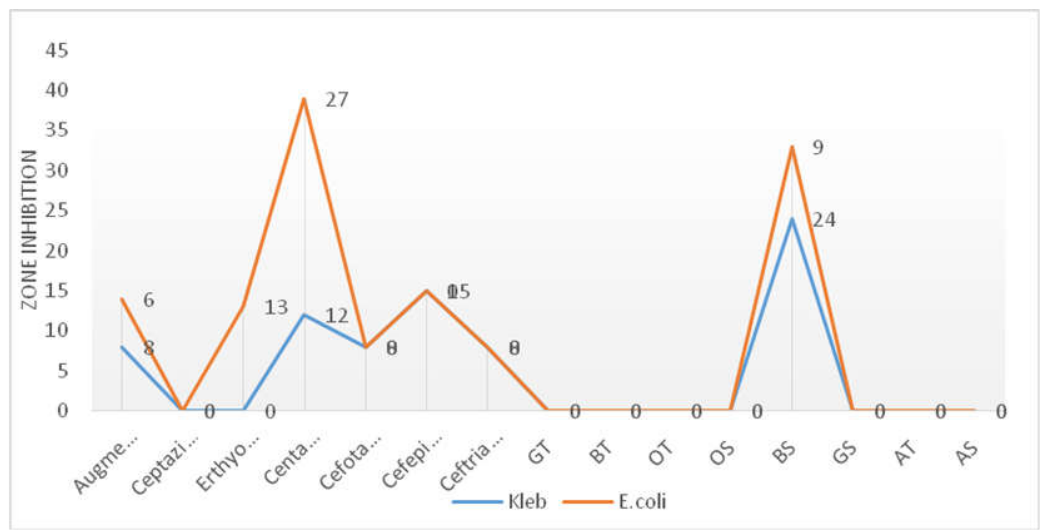

Fig. 8: Comparing fungi metabolites with synthetic antibiotics using Klebsiella spp and Escherichia coli as test organisms

\section{DISCUSSION}

\section{Morphological identification of the soil fungi}

There have been several reviews on successful methods of isolating fungi from nature. There are several fungal species distributed in soil, plant debris, etc. The soil acts as a growth media for fungi in vaious pats of the globe [10]. Many researches have focused on the prevalence and isolation of keratinophilic fungi from soil samples [11-13]. Recently, saprophytic fungi have also been isolated from soil as it causes diseases with people with low immunity [14].

Soil is the most essential source for the isolation of fungi [15]. For example, species belonging to the genera Aspergillus, Penicillium and Mucor have been isolated from roots of soils in different agircultural sites. The results of another study conducted [16] showed that several factors such as $\mathrm{pH}$, organic carbon, moisture conmtent, sulphur, nitrogen and potassium has great effects on soil microbial diversity $[17,18]$. However, these microbes have great potentials applications in drug discovery.

\section{Antimicrobial susceptibility test}

The need for new and more effective antibiotics is on the rise as cases of antibiotic resistance strains of pathogens are increasing daily. This needs have been met for decades largely by the production of semi-synthetic product from the natural product, but in recent years, advancement in technology have refocused researchers in the discovery of natural product and antibiotics from microbial sources, this include finding new antibiotics from older ones as well as from new sources. This breakthrough has ledin the discovery of new antibiotics very novel mechanisms of action capable of forming the bases of new antibiotic classes useful in treating pathogens. Fungi are known for their characteristics of synthesizing a wide range of bioactive metabolites [9]. Naturally occurring antibiotics are mostly isolated from soil microbes. These antiboitics substances contribute vital roles in their establishment on (rhizoplane) and around (rhizosphere) the roots of plants Therefore, to achieve novel compounds, search are to be made from soil microbes; such isolates are potential sources of natural products that posseses secondary metabolites and enzymes [20-22]

\section{CONCLUSION}

In conclusion, the continous exploration of soil microbes, which are reservoirs of drug for the development of novel products is essential for new drug discovery and development. In addition, biomolecules from natural products which are eco-friendly have proven to be abundantly available for utilization as active bio-products against infectious agents of health concern.

\section{ACKNOWLEDGEMENT}

The authors are thankful to Tertiary Education Trust Fund (TETFUND) for providing materials to execute this work and also to the management of LAUTECH, Ogbomoso for the provision of Laboratory space. Thank you.

\section{AUTHORS CONTRIBUTIONS}

The authors have contributed equally in preparing the work, collation of data and writing of the manuscript. 


\section{CONFLICT OF INTERESTS}

Authors don't have any conflict of interest in any way.

\section{REFERENCES}

1. Baron S. Introduction to mycology. 4th Ed. Galveston: University of Texas Medical Branch; 1996.

2. Feofilova EP. The kingdom fungi: heterogeneity of physiological and biochemical properties and relationships with plants, animals, and prokaryotes. Appl Biochem Microbiol 2001;37:124-37.

3. Meyer V. Genetic engineering of filamentous fungi-progress, obstacles and future trends. Biotechnol Adv 2008;26:177-85.

4. Karthikeyan P, Kanimozhi K, Senthilkumar G, Panneerselvam A Ashok G. Optimization of enzyme production in Trichoderma viride using carbon and nitrogen source. Int J Curr Microbiol Appl Sci 2014;3:88-95.

5. Velisek J, Cejpek K. Pigments of higher fungi-a review. Czech J Food Sci 2011;29:87-102

6. Kramer R, Abraham WR. Volatile sesquiterpenes from fungi: what are they good for? Phytochem Rev 2012;11:15-37.

7. Sneath PHA, Sokal RR. Numerical taxonomy. Freeman San Francisco 2010;185:878-82.

8. Tamura K, Nei M, Kumar S. Prospects for inferring very large phylogenies by using the neighbor-joining method. Proc Nat Acad Sci USA 2004:101:11030-5.

9. Kumar S, Stecher G, Li M, Knyaz C, Tamura K. MEGA X: molecular evolutionary genetics analysis across computing platforms. Mol Biol Evolution 2018;35:1547-9.

10. Devi MC, Kumar MS. Isolation and screening of lignocellulose hydrolytic saprophytic fungi from dairy manure soil. Ann Biol Res 2012;3:1145-52.
11. Yazdanparast SA, Dargahi H, Shahrokhi S, Farahani RH. Isolation and investigation of keratinophilic fungi in the parks of municipality districts of Tehran. Thrita 2013;2:2-5.

12. Zarrin M, Haghgoo R. Survey of keratinophilic fungi from soils in Ahvaz, Iran. Jundishapur J Microbiol 2011;4:191-4.

13. Kachuei R, Emami M, Naeimi B, Diba K. Isolation of keratinophilic fungi from soil in isfahan province, Iran. J Myco Med 2012;22:8-13.

14. Shoham S, Levitz SM. The immune response to fungal infections. Br J Haematol 2005;129:569-82.

15. Hara Kishore K, Misra S, Chandra DR, Prakash KVVR, Murty US Antimicrobial efficacy of secondary metabolites from Glomerella cingulata. Braz J Microbiol 2007;38:150-5.

16. Yamaguchi. Trichothecene 3-0-acetyltransferase protects both the producing organism and transformed yeast from related mycotoxins. Cloning and characterization of Tri101. J Biol Chem 1998;273:1654-61.

17. Sharma MS, Raju NS. Frequency and percentage occurrence of soil mycoflora in different crop fields at HD Kote of Mysore district. Inter J Environ Sci 2013;3:1569-76.

18. Yu C, Lv DG, Qin SJ, Du G, Liu GC. Microbial flora in Cerasus sachalinensis rhizosphere. J Appl Ecol 2007;18:2277-81.

19. Beckmann AM, Barrow RA. Fungal metabolites as pharmaceuticals. Aust J Chem 2014;67:827-43.

20. Hunter Cevera JC, Belt A. Isolation of cultures. In: Demain AL Davies JE. (Eds.) Manual of industrial microbiology and biotechnology. 2nd Ed. ASM Press: Washington DC; 1999.

21. Gaddeyya G, Niharika PS, Bharathi P, Kumar PKR. Isolation and identification of soil mycoflora in different crop fields at Salur Mandal. Adv Appl Sci Res 2012;3:2020-6.

22. Dufosse L, Fouillaud M, Caro Y. Filamentous fungi are largescale producers of pigments and colorants for the food industry. Curr Opin Biotechnol 2014;26:56-61. 